\title{
The Drosophila cell adhesion molecule Neuroglian regulates Lissencephaly-I localisation in circulating immunosurveillance cells Michael J Williams
}

Address: Institute of Biological and Environmental Sciences, University of Aberdeen, Tillydrone Avenue, Aberdeen AB24 2TZ, UK

Email: Michael J Williams - m.j.williams@abdn.ac.uk

Published: 25 March 2009

BMC Immunology 2009, 10:17 doi:10.1186/1471-2172-10-17
Received: 4 September 2008

Accepted: 25 March 2009

This article is available from: http://www.biomedcentral.com/147I-2172/10/17

(C) 2009 Williams; licensee BioMed Central Ltd.

This is an Open Access article distributed under the terms of the Creative Commons Attribution License (http://creativecommons.org/licenses/by/2.0), which permits unrestricted use, distribution, and reproduction in any medium, provided the original work is properly cited.

\begin{abstract}
Background: When the parasitoid wasp Leptopilina boulardi lays its eggs in Drosophila larvae phagocytic cells called plasmatocytes and specialized cells known as lamellocytes encapsulate the egg. This requires these circulating immunosurveillance cells (haemocytes) to change from a nonadhesive to an adhesive state enabling them to bind to the invader. Interestingly, attachment of leukocytes, platelets, and insect haemocytes requires the same adhesion complexes as epithelial and neuronal cells.

Results: Here evidence is presented showing that the Drosophila LI-type cell adhesion molecule Neuroglian $(\mathrm{Nrg})$ is required for haemocytes to encapsulate $L$. boulardi wasp eggs. The amino acid sequence FIGQY containing a conserved phosphorylated tyrosine is found in the intracellular domain of all LI-type cell adhesion molecules. This conserved tyrosine is phosphorylated at the cell periphery of plasmatocytes and lamellocytes prior to parasitisation, but dephosphorylated after immune activation. Intriguingly, another pool of $\mathrm{Nrg}$ located near the nucleus of plasmatocytes remains phosphorylated after parasitisation. In mammalian neuronal cells phosphorylated neurofascin, another LI-type cell adhesion molecule interacts with a nucleokinesis complex containing the microtubule binding protein lissencephaly-I (LisI) [I]. Interestingly in plasmatocytes from $\mathrm{Nrg}$ mutants the nucleokinesis regulating protein Lissencephaly-I (LisI) fails to localise properly around the nucleus and is instead found diffuse throughout the cytoplasm and at unidentified perinuclear structures. After attaching to the wasp egg control plasmatocytes extend filopodia laterally from their cell periphery; as well as extending lateral filopodia plasmatocytes from $\mathrm{Nrg}$ mutants also extend many filopodia from their apical surface.

Conclusion: The Drosophila cellular adhesion molecule Neuroglian is expressed in haemocytes and its activity is required for the encapsulation of $L$. boularli eggs. At the cell periphery of haemocytes Neuroglian may be involved in cell-cell interactions, while at the cell centre Neuroglian regulates the localisation of the nucleokinesis complex protein lissencephaly-I.
\end{abstract}

\section{Background}

When the morphology of Drosophila haemocytes is compared, three types of cells can be identified: plasmatocytes, lamellocytes and crystal cells. Plasmatocytes resemble the mammalian monocyte/macrophage lineage and are involved in the phagocytosis or encapsulation of invading pathogens [2,3]. Lamellocytes are larger than the other haemocytes, are rarely seen in healthy larvae and seem to be specialized for the encapsulation of invading pathogens $[4,5]$. Crystal cells rupture to secrete components of 
the phenol oxidase cascade, involved in melanisation of invading organisms, wound repair and coagulation [6-8]

Endoparasitic wasps from the Hymenoptera family are known to parasitize Drosophila larvae. Once the invader is recognized as foreign circulating plasmatocytes somehow adhere and spread around the egg. After spreading the plasmatocytes form cellular junctions between the cells effectively separating the egg from the larval open circulatory system (hemoceol) [9,10]. Following plasmatocyte adherence and spreading, lamellocytes recognize the plasmatocytes surrounding the egg, and finally the capsule is melanised due to crystal cell rupture [9-11]. From these events it is obvious that adhesion and cell shape change are essential parts of the Drosophila's cellular immune response against parasitoid wasp eggs.

Circulating immune surveillance cells need to remain mobile until they receive the correct signals to become adherent. In the case of Drosophila larvae, haemocytes change from non-adhesive circulating cells to adhesive non-circulating cells after parasitisation. Evidence is mounting that during attachment or encapsulation events leukocytes, platelets, and insect haemocytes use the same adhesion complexes as epithelial and neuronal cells $[10,12-19]$. In platelets the mammalian homolog of Neuroglian, L1-Cam is necessary for platelet-platelet interactions [20]. Furthermore in the tobacco hornworm Manduca sexta the L1-Cam family member Neuroglian has been shown to interact with integrins during immune encapsulation responses $[17,18]$. Because of these results I decided to look at the involvement of Neuroglian in the Drosophila cellular immune response against eggs from the parasitoid wasp Leptopilina boulardi.

\section{Results}

\section{Neuroglian cellular localization}

To begin to elucidate if Drosophila Neuroglian (Nrg) was involved in the cellular immune response haemocytes were bled from parasitized control larvae $\left(w^{1118}\right)$ approximately 40 hours after parasitisation and co-stained with anti- $\alpha$-Tubulin and anti-Nrg antibodies [21]. In both plasmatocytes and lamellocytes bled from parasitized control larvae Nrg was expressed at the plasma membrane, and accumulated in filopodia at the cell periphery (Figure 1, arrows).
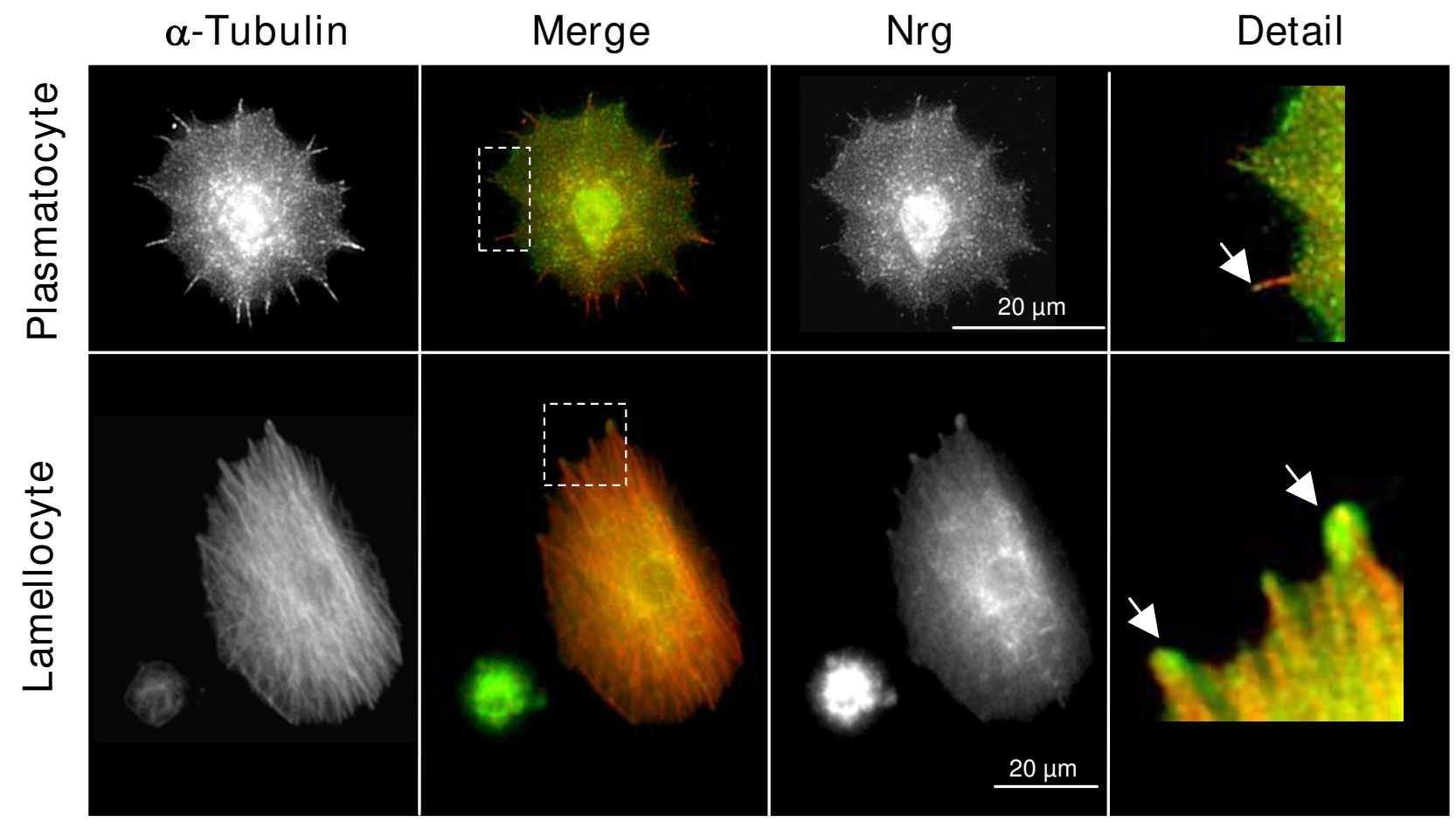

Red $=\alpha$-Tubulin Green $=$ Nrg

Figure I

Nrg expressed in plasmatocytes and lamellocytes. Plasmatocytes and lamellocytes bled from control larvae 38-40 h post-parasitisation by L. boulardi G486 and co-stained with anti-Tubulin (red) and anti-Nrg (green). In the detail figure of plasmatocytes and lamellocyte arrows indicate that $\mathrm{Nrg}$ protein is enriched at the tips of some filopodia. 
$\mathrm{Nrg}$ has two splice forms one of which, $\mathrm{Nrg} 180$, is specifically expressed in the nervous system [22]. To make sure that the Nrg protein expressed in haemocytes is not Nrg180, haemocytes bled from non-parasitized larvae, as well as from larvae 24 and $40 \mathrm{~h}$ post-parasitisation were stained with a mouse monoclonal antibody that specifically recognizes $\operatorname{Nrg}^{180}$ [22]. No staining was observed in any of the haemocytes (data not shown), showing that the $\mathrm{Nrg}$ expressed in haemocytes is not $\mathrm{Nrg}^{180}$.

\section{Nrg-FIGQY dephosphorylated after parasitisation}

Neuroglian belongs to the L1-family of cellular adhesion molecules, along with mammalian L1, Neurofascin, NRCAM, NgCAM and Caenorhabditis elegans LAD-1 $[21,23,24]$. All L1 family members have the conserved amino acid sequence FIGQY containing a tyrosine phosphorylation site in their intracellular domain. Dephosphorylation of the FIGQY tyrosine allows L1-type cellular adhesion molecules (CAMs) to interact with ankyrin, and through ankyrin to interact with the spectrin cortical-actin cytoskeleton [25]. Using an antibody raised against phospho-FIGQY [26] it was evident that in plasmatocytes bled from non-parasitized control larvae the conserved NrgFIGQY tyrosine was phosphorylated at the cell periphery (Figure 2B and 2D) and also at sites near the nucleus (Figure $2 \mathrm{C}$ ). In plasmatocytes bled from larvae $40 \mathrm{~h}$ post-parasitisation there was virtually no phospho-tyrosine observed at the cell periphery (Figure 2E and $2 \mathrm{H}$ ), while Nrg was still phosphorylated at sites near the cell centre (Figure 2G). In lamellocytes it was not as obvious that $\mathrm{Nrg}$ was phosphorylated at the plasma membrane in non-parasitized larvae (Figure 2J and $2 \mathrm{~L}$ ), though it was evident that there was less phosphorylation $40 \mathrm{~h}$ after parasitisation (Figure $2 \mathrm{~N}$ and $2 \mathrm{P}$ ). There was never any accumulated phospho-Nrg observed near the cell centre of lamellocytes bled from either non-parasitized or parasitized control larvae (Figure 2K and 2O).

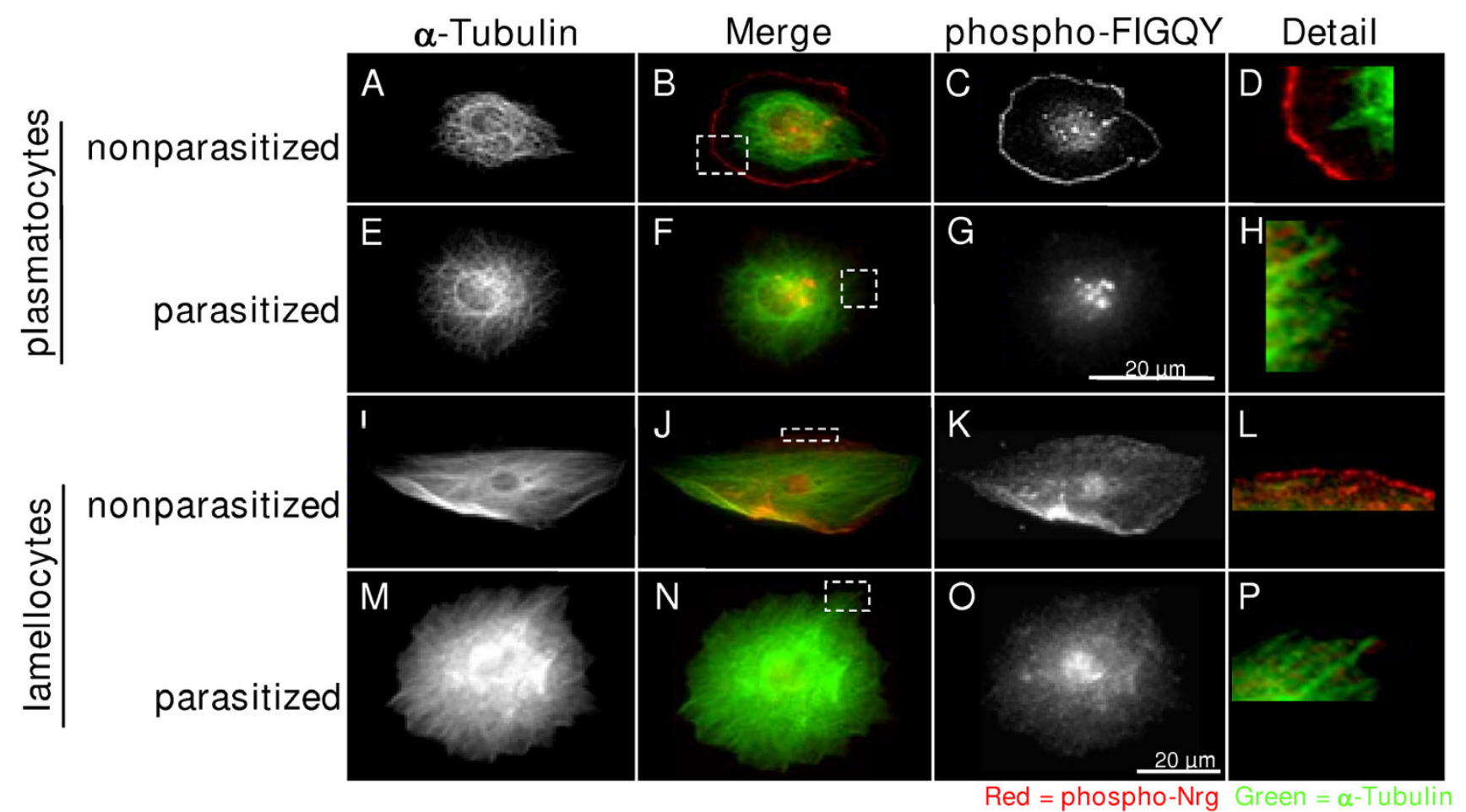

Figure 2

Nrg-FIGQY phosphorylation regulated by immune activation (A-H) Plasmatocytes bled from non-parasitized control larvae or 38-40 h post-parasitisation, and stained with anti- $\alpha$-Tubulin (A, E) and anti-phospho-FIGQY (C, G). (B) Merge of A and C, square denotes area shown in D. (D) Detail of the plasma membrane of a plasmatocyte from a non-parasitized larva. (F) Merge of $E$ and $G$, square denotes area shown in $H$. $(H)$ Detail of the plasma membrane of a plasmatocyte from a parasitized larva. (I-P) Lamellocytes bled from non-parasitized control larvae or $38-40 \mathrm{~h}$ post-parasitisation, and stained with anti-Tubulin $(\mathrm{I}, \mathrm{M})$ and anti-phospho-FIGQY $(\mathrm{K}, \mathrm{O})$. (J) Merge of I and K, square denotes area shown in L. (L) Detail of the plasma membrane of a lamellocyte from a non-parasitized larva. $(N)$ Merge of $M$ and $O$, square denotes area shown in P. (P) Detail of the plasma membrane of a plasmatocyte from parasitized larva. 


\section{Neuroglian required for Lis I perinuclear localization} In rat neuroblastoma cells the phosphorylated FIGQYdomain of neurofascin is bound by doublecortin [1]. Doublecortin ( $\mathrm{Dcx}$ ) is a microtubule binding protein that when mutated causes a type of neuronal migration disorder known as X-linked lissencephaly [27]. Doublecortin has been shown to interact with another microtubule associated protein known as lissencephaly-1 (Lis1) [28], and together they are involved in regulating the movement of the nucleus of neuronal cells during migration [29]. Phosphorylation the phospho-FIGQY-Nrg near the nucleus of plasmatocytes could allow Nrg to interact with a Dcx-Lis1 complex. To test this possibility, plasmatocytes from non-parasitized control, $\mathrm{Nrg}^{\mathrm{G} 00305}$ mutant larvae, or larvae overexpressing an Nrg RNAi construct (UAS-NrgIR, from now on referred to as $N r g^{I R}$ ) specifically in haemocytes using the haemocyte-specific driver Hemese-Gal4 (He-Gal4) [30], were stained for Lis 1 expression using an antibody raised against Human Lis1. Null mutations of $\mathrm{Nrg}$ are homozygous lethal, so the $\mathrm{Nrg} G 00305$ allele which survives to adulthood was chosen for this study [31]. $N r g G 00305$ may be a weak hypomorph with enough function to survive embryogenesis [32]. In plasmatocytes bled from control larvae Lis1 was observed surrounding the nucleus and at what could be the centrioles (Figure 3A, arrows, and 3B). In $\mathrm{Nrg}^{\mathrm{G00305}}$ or $\mathrm{Nrg}^{\mathrm{IR}} ; \mathrm{He}$-Gal4 plasmatocytes less Lis 1 was observed surrounding the nucleus, and its expression looked more diffuse throughout the cytoplasm than in controls (see Figure 3A, merged image). In these same cells Lis1 protein was enriched at what appeared to be perinuclear centriole-like structures that were not observed in control plasmatocytes (Figure 3A, arrowheads). To make sure that the antibody was specifically recognizing Drosophila Lis1 we crossed He-Gal4 to flies overexpressing a Lis1 RNAi construct (UAS-Lis $1^{I R}$ ). Lis 1 expression was significantly reduced in $\mathrm{He}$-Gal4/UASLis $1^{I R}$ haemocytes (Figure 4).

To test if the Lis1-localised structures observed in $\mathrm{NrgG00305}$ and $\mathrm{Nrg}^{\mathrm{IR}} ; \mathrm{He}$-Gal4 plasmatocytes were centrioles plasmatocytes were co-stained with anti-Lis1 and anti- $\gamma$-tubulin. In control plasmatocytes Lis 1 and $\gamma$-tubulin co-localised at the centrioles (Figure 5A, arrowheads). In plasmatocytes recovered from $\mathrm{Nrg}^{\mathrm{GO00305}}$ or $\mathrm{Nrg}^{I R} ; \mathrm{He}$ Gal4 mutant larvae Lis 1 and $\gamma$-tubulin still co-localised at the centrioles (Figure 5A, arrowheads). Interestingly, $\gamma$ tubulin did not localise to the Lis1 enriched structure observed in $\mathrm{Nrg}^{\mathrm{G} 00305}$ or $\mathrm{Nrg}{ }^{I R} ; \mathrm{He}$-Gal4 plasmatocytes (Figure 5A, arrows). In lamellocytes bled from parasitized control, $\mathrm{Nrg}{ }^{\mathrm{G00305}}$ or $\mathrm{Nrg}{ }^{I R} ; \mathrm{He}$-Gal4 larvae Lis1 expression was localised around the nucleus (Figure 5B). Interestingly, in lamellocytes from $\mathrm{Nrg}^{\mathrm{G00305}}$ or $\mathrm{NrgIR} ; \mathrm{He}$-Gal4 larvae Lis1 protein was still tightly localised around the nucleus (Figure 5B, and data not shown). This result may not be surprising as lamellocytes contain polytene chro- mosomes, are fully differentiated, and no centrioles were observed (data not shown).

\section{In NrgG00305 Nrg still present at plasma membrane}

The P-element insert that created the NrgG00305 allele inserted into the intron prior to the exon containing the FIGQY amino acid sequence (Figure 6A) [31]. The design of the P $\{$ PTT-GA $\}$ P-element allows for the incorporation of green fluorescent protein (GFP) into the open reading frame of $\mathrm{Nrg}$, creating a chimeric protein [31]. To see if the insertion of GFP in the intracellular domain affected Nrg expression, Control and $\mathrm{Nrg}^{\mathrm{G} 00305}$ haemocytes were collected form non-parasitized third instar larvae and stained for Nrg expression. No difference in Nrg expression or localisation was observed when $\mathrm{NrgG00305}$ haemocytes were compared to controls (Figure 6B). Furthermore, UAS-NrgIR;He-Gal4 haemocytes Nrg expression was significantly reduced compared to control cells (Figure 6B). Next, to test if the insertion of GFP in the intracellular domain affected Nrg-FIGQY phosphorylation, haemocytes from non-parasitized control and NrgG00305 mutants were stained with anti-phospho-FIGQY antibodies. In control and $\mathrm{Nrg}^{\mathrm{G} 00305}$ mutant plasmatocytes phoshoFIGQY-Nrg was present at the cell periphery (Figure 6C) and near the nucleus (Figure 6C, arrows). Plasmatocytes bled from UAS-NrgIR;He-Gal4 larvae had less phoshoFIGQY-Nrg at the cell periphery, and very little phoshoFIGQY-Nrg was observe near the cell centre. From this result I conclude that the GFP insert in the intracellular domain does not affect Nrg localisation or the phosphorylation of the FIGQY conserved tyrosine.

\section{Neuroglian needed for encapsulation}

An encapsulation assay was performed on larvae parasitized by the avirulent $L$. boulardi wasp strain G486. When the avirulent wasp strain G486 parasitizes larvae a darkened cellular capsule is visible in the hemoceol 30-40 h later. While $81 \%$ of $\mathrm{Nrg}^{\mathrm{G00305}}$ heterozygous mutant larvae properly encapsulated and melanised L. boulardi eggs, $N r g \mathrm{G00305}$ homozygous larvae never properly encapsulated the wasp egg (Figure 7A). Similar to NrgG00305 homozygous mutants, none of the larvae expressing $\mathrm{Nrg}$ RNAi in haemocytes properly encapsulated the wasp egg (Figure 7A).

In the encapsulation assay a larva is considered to have a defective cellular immune response when the wasp egg is not melanised. Melanisation is the final event in encapsulation, so this assay is not able to define the actual defect during the encapsulation process. To gain a better understanding of when the activity of $\mathrm{Nrg}$ is required during encapsulation, wasp eggs were recovered from either control or homozygous $\mathrm{Nrg}^{\mathrm{G00305}}$ mutant larvae at various times after parasitisation and stained for haemocyte specific markers. In general plasmatocytes attach to the wasp 

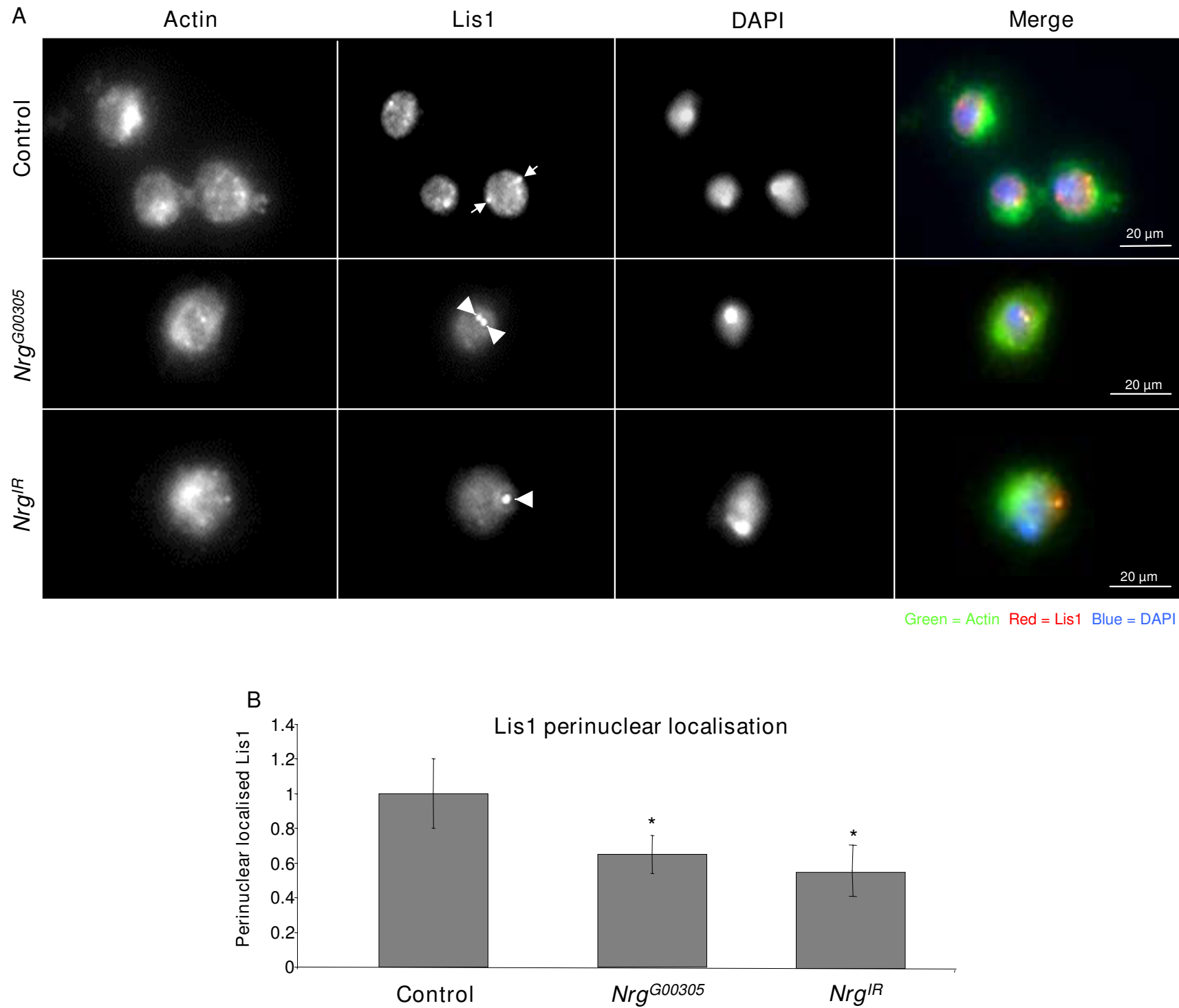

Figure 3

Lis I protein mislocalised in Nrg mutants. (A) Haemocytes were bled from wandering third instar control ( $w^{1 / 18}$ ), $\mathrm{Nrg}{ }^{G 00305}$, or UAS-NrgIR;He-Gal4 larvae and stained for LisI expression (red) and actin (green), nuclei were visualised by DAPI staining (blue). In control cells Lis I was observed tightly associated with the nucleus and at centriole-like structures also tightly associated with the nucleus (arrows). In plasmatocytes from Nrg G00305 or UAS-NrgIR;He-Gal4 larvae Lis I was more diffuse throughout the cytoplasm, and in many cells Lis I expression was enhanced at centriole-like structures (arrowheads). (B) Lis I perinuclear expression levels. Haemocytes were bled from control, NrgG00305 or UAS-Nrg;R;He-Gal4 wandering third instar larvae. The haemocytes were stained for Lis I expression. ImageJ was used to measure fluorescence intensity of Lis I staining surrounding the nucleus of at least 75 haemocytes from three different larvae. Asterisks indicate a significant difference from control cells. (Student's $t$-test, $P<0.0 \mathrm{I}$ ).

egg between 6-24 h after the egg is laid in the larval hemoceol [11]. To look at plasmatocytes during the encapsulation process wasp eggs were dissected from larvae 22-24 $h$ post-parasitisation and stained for the plasmatocyte specific protein Nimrod [33,34]. By 22-24 h post-parasitisation wasp eggs recovered from control larvae ( $w^{1118}$ or
$\mathrm{Nrg} \mathrm{G00305} /+$ ) were completely encapsulated by plasmatocytes that had spread around the chorion (Figure $7 \mathrm{~B}$ ). Plasmatocytes that had not made cell-cell contacts sent out filopodia from the cell periphery towards other plasmatocytes (Figure 7B, inset). Eggs recovered from homozygous $\mathrm{Nrg}^{\mathrm{G} 00305}$ mutants also had plasmatocytes 


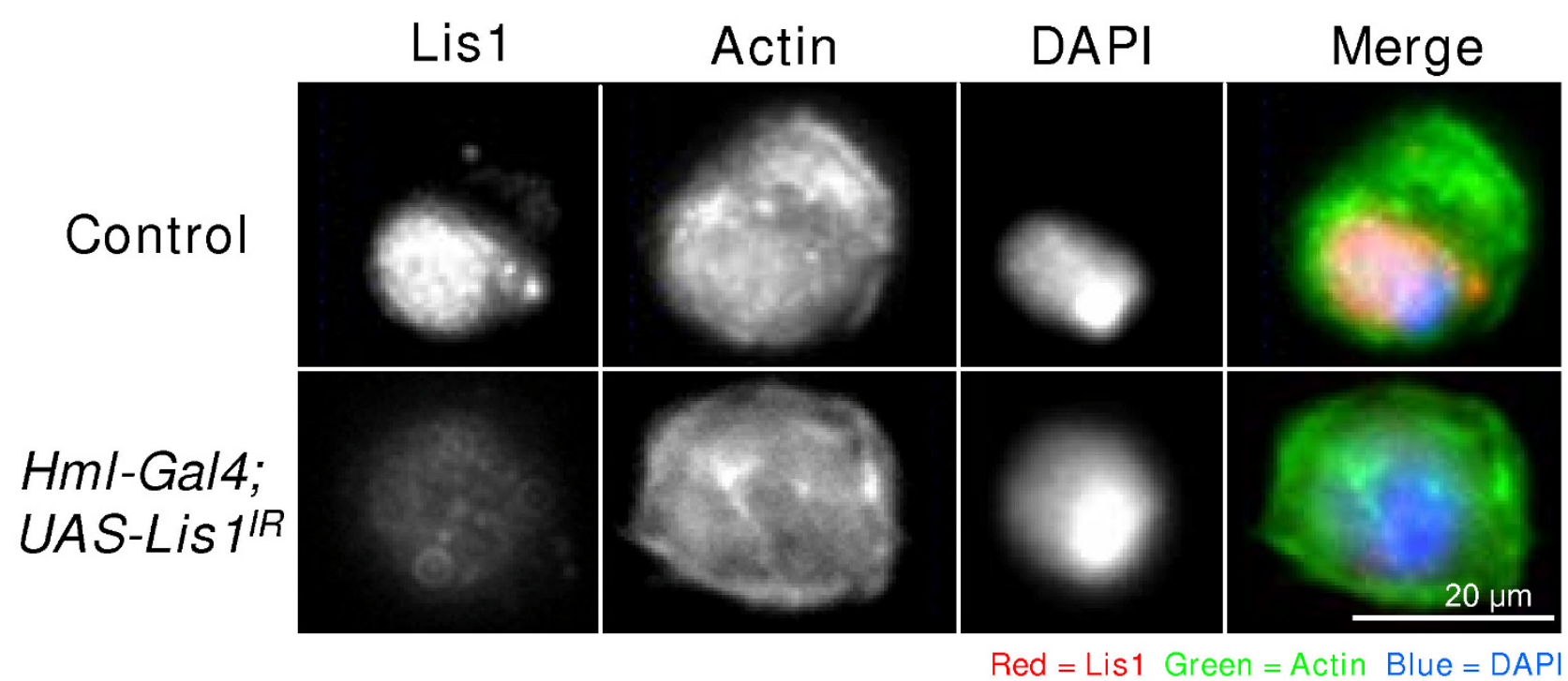

Figure 4

Haemocytes were bled from non-parasitized control and He-GA4/UAS-Lis IIR larvae and stained for Lis I (red) and Actin (green), nuclei were visualised by DAPI staining (blue). Size bar indicates $20 \mu \mathrm{m}$.

attached to the wasp egg that had spread on the chorion. In most instances fewer plasmatocytes were attached to the egg than in control larvae and in some cases almost no plasmatocytes were attached (Figure 7C and data not shown). Nrg mutant plasmatocytes attached to the wasp egg also extended filopodia laterally from their cell periphery (Figure 7C, inset); yet unlike controls, NrgG00305 mutant plasmatocytes projected many filopodia from
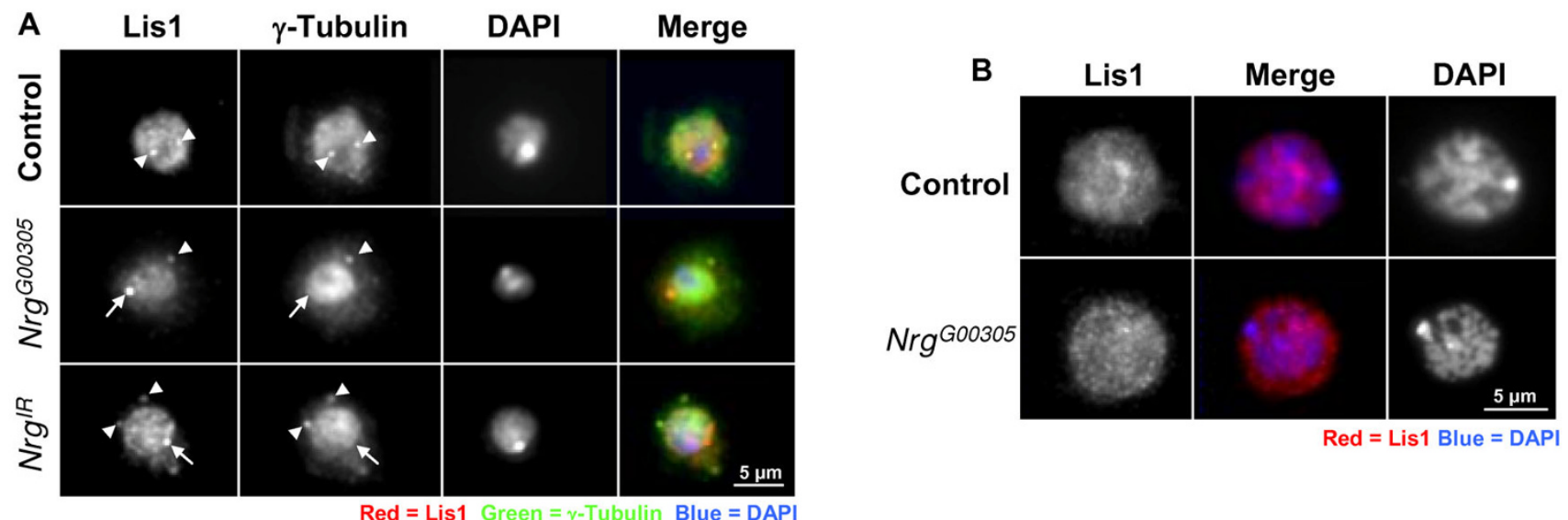

Figure 5

(A) Haemocytes were bled from non-parasitized control larvae and stained for Lis I (red) and $\gamma$-Tubulin (green), nuclei were visualised by DAPI staining (blue). Arrowheads indicate co-localisation of Lis I and $\gamma$-Tubulin at the centrioles. Open-arrows indicate LisI specific expression seen in NrgG00305 and UAS-NrgIR;He-Gal4 plasmatocytes. (B) Lamellocytes from parasitized control, and NrgG00305 larvae stained for Lis (red) and the nuclei were visualised by DAPI staining (blue). 


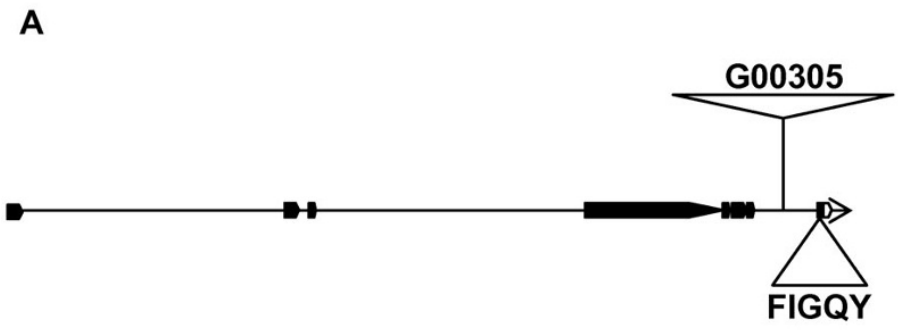

C

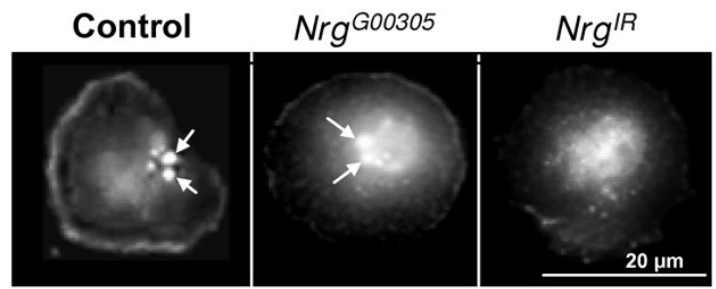

B

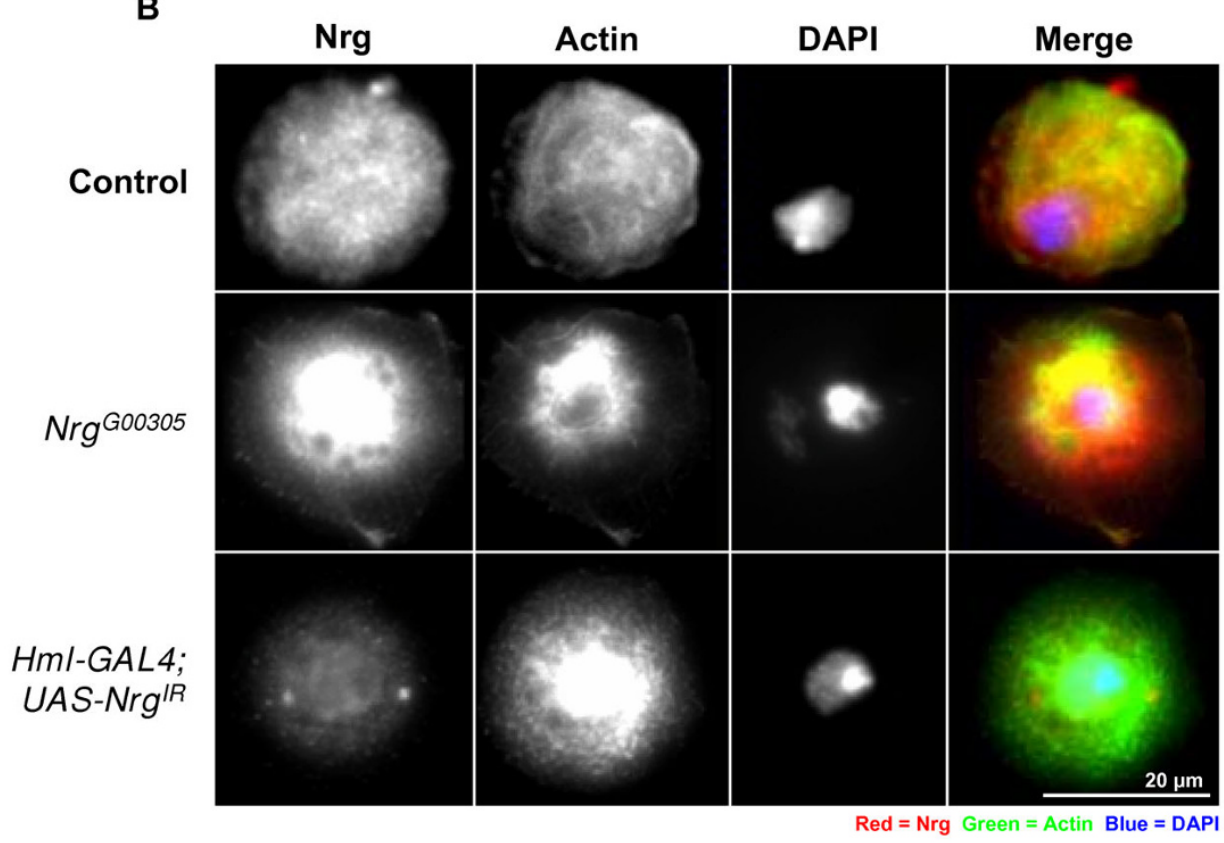

\section{Figure 6}

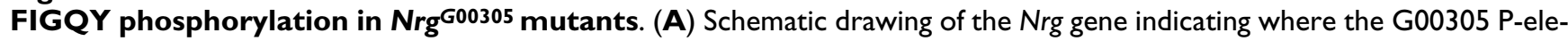
ment is inserted. (B) Haemocytes were bled from non-parasitized control NrgG00305 and UAS-Nrg; ; He-Gal4 wandering third instar larvae and stained for Nrg expression. Nrg (red), Actin (green), nuclei were visualised by DAPI staining (blue). (C) Haemocytes were bled from non-parasitized control Nrg G00305 and UAS-Nrg/R;He-Gal4 wandering third instar larvae and stained for phospho-FIGQY-Nrg. Arrows indicate phosphorylated Nrg near the nucleus.

38-40 h post-parasitisation eggs recovered from control larvae were completely surrounded by fully spread lamellocytes (Figure 7D). In most $\mathrm{Nrg}$ G00305 homozygous mutant larvae no lamellocytes were attached to wasp eggs, but in a few larvae a couple of lamellocytes were attached to the egg (Figure 7E, and data not shown).

\section{Discussion}

The Drosophila cellular adhesion molecule Neuroglian is expressed in haemocytes and its activity is required for them to properly encapsulate eggs from the parasitiod wasp L. boulardi. It is possible that Nrg plays multiple roles when plasmatocytes adhere and spread on wasp eggs. At the cell periphery it could be involved in cell-cell interac- tions, while at the cell centre Nrg may regulate the localisation of a lissencephaly-1 containing complex.

In Drosophila larvae reduced Neuroglian activity caused an impairment of haemocyte adhesion to the wasp egg and reduced cell-cell interactions between plasmatocytes and lamellocytes. Similar to encapsulation events in $M$. sexta these two steps of encapsulation may require heterophilic interaction between $\mathrm{Nrg}$ and integrins, $\mathrm{Nrg}$ homophilic binding, or both $[17,18]$. In addition, both events may be accompanied by dephosphorylation of FIGQY to allow interaction of Nrg with ankyrin. Nrg may become dephosphorylated at the cell periphery to allow it to interact with Ankyrin protein and thus to the spectrin cortical-cytoskeleton $[25,26]$. Nrg has been localised to 

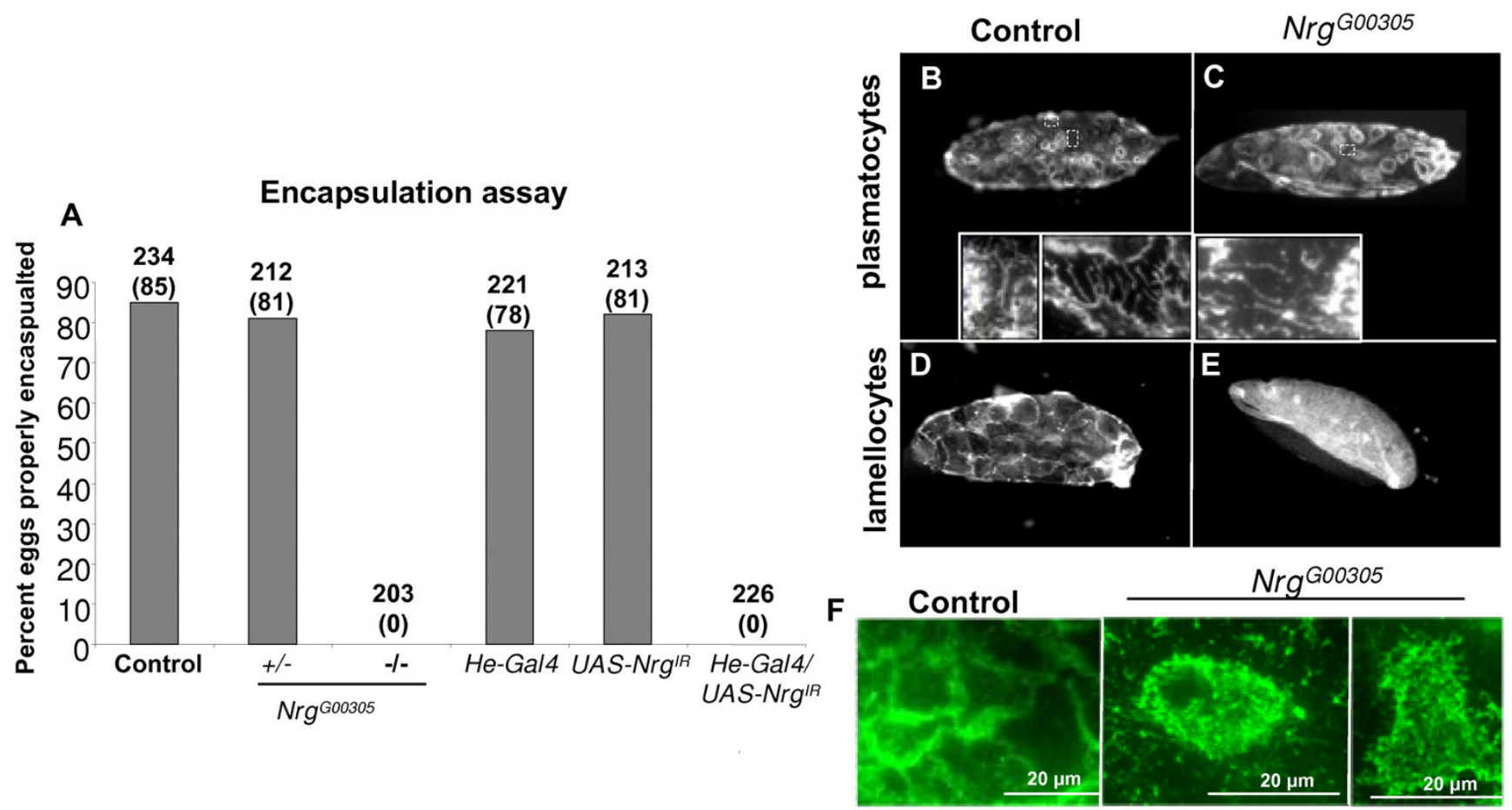

\section{Figure 7}

Nrg necessary for proper encapsulation of $L$. boulardi eggs (A) Encapsulation capacities of $\mathrm{Nrg}$ mutant larvae in response to parasitisation by $L$. boulardi G486. [(Number of larvae with properly melanized wasp eggs/number of parasitized larvae) $\times$ 100)]. Numbers above the bars indicate the number of wasp parasitized larvae examined, numbers in parentheses indicate percentage of larvae that properly encapsulated the L. boulardi G486 eggs (B, C) Encapsulated wasp eggs recovered from larvae 22-24 h after parasitisation, and stained with the plasmatocyte specific protein Nimrod. (B) Control ( $w^{\prime \prime l}$ l 8 ) and (C) Nrg ${ }^{G 00305}$ homozygous mutants. (D, E) Haemocytes 38-40 h post-parasitisation stained with the LI lamellocyte specific antibody. (D) Control and (E) NrgG00305 homozygous mutants. (F) Control and Nrg ${ }^{600305}$ plasmatocytes attached to L. boulardi G486 eggs. Unlike controls, plasmatocytes from $\mathrm{Nrg}^{\mathrm{G} 00305}$ extend filopodia from their apical side.

septate junctions in Drosophila embryonic epithelial cells and its activity is necessary for septate junction formation [35]. In the cellular immune response against parasitoid wasp eggs Nrg may be necessary for plasmatocytes to form cellular junctions during the encapsulation response. Once plasmatocytes spread around the wasp egg and make cell-cell contacts they form cellular junctions [9]. These junctions have been described as looking like septate junctions, and at least one septate junction protein, Coracle, has been localised to the cell-cell interactions of plasmatocytes on wasp eggs [10].

In neurons it has been shown that the L1-family member neurofascin interacts with doublecortin and this interaction is necessary for neuronal migration [1]. Doublecortin is a microtubule-associated protein involved in neuronal migration [29], and along with the microtubule array and neurofascin, doublecortin interacts with lissencephaly-1 (LIS1). In both mammalian and Drosophila neurogenesis, Lis-1 is necessary for neuroblast proliferation and migra- tion [36-38]. The doublecortin-Lis 1 interaction is necessary for nucleokinesis during neuronal migration [39]. It is speculated that the interaction with neurofascin may be necessary to anchor the Lis 1 complex to generate the force necessary for nucleokinesis, and without the signal from neurofascin nucleokinesis and cell migration cannot occur $[1,36]$. It may be that phosphorylation of the NrgFIGQY tyrosine at the plasmatocyte cell centre is necessary for Nrg to interact with a Drosophila doublecortin domain (Dcx) containing protein, to allow for nuclear anchoring. In an $\mathrm{Nrg}^{\mathrm{G00305}}$ mutant plasmatocyte Nrg may not be able to interact with a Dcx-domain protein, thus the Dcx-Lis1 complex cannot interact properly with the nuclear membrane and ends up at the unidentified perinuclear centriole-like structures or diffuse in the cytoplasm. There is no obvious homolog of mammalian doublecortin in Drosophila, but there are three proteins that contain Dcx domains, two of which are very similar to other doublecortin domain proteins called doublecortin-like kinase- 1 and -2 in mammals. In a study to define the 
interaction of doublecortin with neurofascin, three amino acids in doublecortin were discovered to be important for this interaction [1]. Interestingly, all three of these amino acids are conserved in the Drosophila doublecortin-like kinase homolog CG17528 and the Dcx-domain protein CG42247, while two are conserved in the doublecortinlike kinase homolog CG10177 (Figure 8A). The possibility that Nrg interacts with one of these Dcx-domain containing proteins to anchor the nucleus during plasmatocyte spreading is currently under investigation. Of further interest is the observation that CG42247 was found to interact with another cell adhesion molecule Echinoid in a yeast-two hybrid screen [40]. Though Echinoid does not contain the FIGQY sequence found in L1family molecules it does contain a similar sequence, FEGEY, in its intracellular domain near the C-terminus (Figure 8B).

In $\mathrm{Nrg}{ }^{\mathrm{G} 00305}$ mutants the FIGQY tyrosine is still phosphorylated yet Lis1 mislocalises in the mutant plasmatocytes. This leads to the possibility that phosphorylation of the FIGQY tyrosine is not sufficient for interaction of Nrg with the Lis1 complex. In the study where neurofascin was shown to interact with doublecortin, it was shown that doublecortin only slightly bound to L1-CAM, and not at all to NRCAM, even though they also contain phosphoFIGQY [1]. There is another conserved tyrosine found in all L1-family members upstream of the FIGQY sequence (Figure 9). This tyrosine is predicted to be phosphorylated in both Neuroglian and neurofascin, but not in L1-CAM or NRCAM. The GFP sequence the NrgG00305 allele is incorporated between these two conserved tyrosines and may disrupt their interaction with the Lis1 complex. Also, even though it was shown that phospho-FIGQY was necessary for the interaction of neurofascin with doublecortin in vivo, it may not be sufficient [1]. Another possibility is insertion of GFP into the Nrg open reading frame may change the conformation of the intracellular domain blocking the interaction of $\mathrm{Nrg}$ with the Lis1 complex even though the conserved FIGQY tyrosine is phosphorylated.

\section{Conclusion}

Most of what we understand about the complex that regulates nucleokinesis comes from studies on neuronal development [29]. Yet, one of the first proteins discovered to regulate nucleokinesis, lissencephely-1/NUDF was discovered in T cells [41]. Leukocytes migrating through interstitial tissues must solve many of the same problems as neurons migrating during development, one of the main problems being nuclear migration. What is not fully understood in neurons, and not studied at all in the cellular immune response, is exactly how the nucleokinesis complex regulates nuclear migration. Here evidence was presented showing that in one subtype of Drosophila circulating immunosurveillance cells a transmembrane molecule, Neuroglian, somehow regulates the localisation of at least one nucleokinesis complex protein, Lis- 1 . What has not been elucidated is the significance of this in nucleokinesis or if this aspect of Neuroglian function is important for cellular immune response function. Still, it is intriguing to speculate that similar to neuronal cells, immunosurveillance cells also use the nucleokinesis apparatus to regulate nuclear migration.

\section{Methods \\ Insects}

Drosophila strains $w^{1118}$ and $\mathrm{Nrg} \mathrm{g}^{\mathrm{G} 00305}$ were obtained from the Bloomington Stock Centre. Hemese-GAL4 driver line has been described previously [30]. UAS-NrgIR strain number 27201 and UAS-Lis ${ }^{I R}$ strain number 6216 were obtained from the Vienna Drosophila RNAi Centre (VDRC). Flies were kept on a standard corn molasses meal diet at between $21-25^{\circ} \mathrm{C}$. The G486 strain of L. boulardi was bred on a $w^{1118}$ stock of D. melanogaster at room temperature using a standard medium. Adult wasps were maintained at room temperature on grape juice agar.

\section{Wasp egg encapsulation assay}

The encapsulation assay was done according to Sorrentino et al., [5]. Briefly, two days before parasitisation the appropriate fly strains were crossed and kept at $21-25^{\circ} \mathrm{C}$. Four or five females of $L$. boulardi G486 were allowed to infest at room temperature for 2 hours, after which the Drosophila larvae were transferred to apple juice plates and left at room temperature for 40-42 hours. After this time the larvae were collected, washed in PBS, and then viewed under a stereomicroscope for the presence of a dark capsule. Larvae in which no dark capsule was observed were dissected in $20 \mu \mathrm{l}$ of PBS to determine if they had been parasitized. Larvae containing eggs from the parasitoid that hadn't darkened by this time were scored as nonencapsulated. Non-parasitized larvae were excluded from the count.

\section{Antibodies and reagents}

Lamellocyte specific mouse monoclonal antibody (L1a) [33] and plasmatocyte specific monoclonal mouse antiNimrod [33,34] were used undiluted, mouse monoclonal antibody anti- $\alpha$-Tubulin (Sigma) was diluted 1:1,000, rabbit polyclonal anti- $\alpha$-Tubulin (Abcam) was diluted $1: 500$, mouse monoclonal anti- $\gamma$-Tubulin (Sigma) was diluted 1:500, rabbit polyclonal anti-phospho-FIGQY was diluted 1:250 [26], mouse monoclonal anti-Nrg 3C1 was diluted 1:1,000 [21,23], rabbit polyclonal anti-Lis1 (Abcam, ab2607) was diluted 1:500, and mouse monoclonal anti-Nrg ${ }^{180}$ (BP104, Developmental Studies Hybridoma Bank) was used undiluted. 
A

Human Dcx

Drosophila CG17528 Dcx Drosophila CG42247 Dcx Drosophila CG10177 Dcx

Human Dcx Drosophila CG17528 Dcx Drosophila CG42247 Dcx Drosophila CG10177 Dcx

Human Dcx Drosophila CG17528 DCX Drosophila CG42247 Dcx Drosophila CG10177 Dcx

Human Dcx Drosophila CG17528 Dcx Drosophila CG42247 Dcx Drosophila CG10177 Dcx

Human Dcx
Drosophila CG17528 Dcx
Drosophila CG42247 Dcx
Drosophila CG10177 Dcx
Human Dcx
Drosophila CG17528 Dcx
Drosophila CG42247 Dcx
Drosophila CG10177 Dcx
Human Dcx
Drosophila CG17528 Dcx
Drosophila CG42247 Dcx
Drosophila CG10177 Dcx

B

Figure 8

Sequence alignments (A) Sequence alignment of three Drosophila proteins containing Dcx-domains and human doublecortin. Underlined sequences indicated Dcx domains, filled circles indicate the three amino acids shown to be necessary for doublecortin to interact with neurofascin. (B) Sequence alignment comparing the FIGQY domains of Human LICAM, Human Neurofascin and Drosophila Neuroglian with Drosophila Echinoid. 


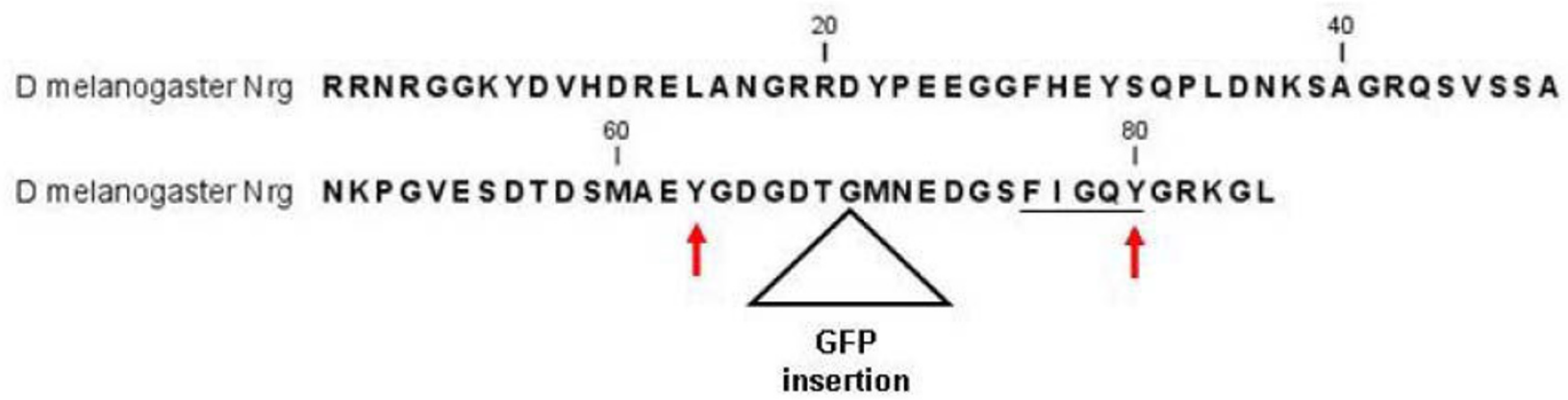

Figure 9

Neuroglian 167 intracellular domain showing the conserved FIGQY sequence (underline) and the two predicted tyrosine phosphorylation sites (arrows). The location of the inserted GFP sequence is also indicated. Phosphorylation of the conserved tyrosines was predicted using the Netphos 2.0 program.

\section{Immunofluorescence}

Wasp egg staining

For lamellocyte monoclonal antibody (L1a) and the plasmatocyte specific monoclonal antibody (P1a), wasp eggs were bled from larvae, into $20 \mu$ l of phosphate buffered saline (PBS), and allowed to attach to a glass slide (SM011, Hendley-Essex, Essex, UK) for 5 minutes at room temperature. Staining and analysis were done according Williams et al., [10].

\section{Circulating haemocyte staining}

For all haemocyte antibody staining, haemocytes were bled from a larvae into $20 \mu \mathrm{l}$ of PBS, and allowed to attach to a glass slide (SM-011, Hendley-Essex, Essex, UK) for 1 hour. Staining and analysis were done according to Williams et al., [42]. Cells were visualized using a Zeiss Axiovert $200 \mathrm{M}$ epifluorescent microscope and digital pictures were taken with a Hamamatsu C4742-80-12AG video unit, controlled by the Simple PCI 6.1 program (Hamamatsu). ImageJ (NIH) was used for digital editing. ImageJ was used to measure fluorescent intensity.

\section{Acknowledgements}

I would like to thank Dr M. Hortsch and. V. Bennett for their generous gifts of Neuroglian and phospho-FIGQY antibodies respectively. Furthermore, I would like to thank Istvan Ando for his kind gift of the anti-LI lamellocyte and anti-Nimrod plasmatocyte antibodies. I would also like to thank the Developmental Studies Hybridoma Bank developed under the auspices of the NICHD and maintained by the University of lowa for providing the BPI04 mouse monoclonal antibody. This research was supported in part by funds provided to Michael Williams by The Royal Society. This research was also partially funded by Dan Hultmark's grants from the Swedish Research Council and the Swedish Cancer Society. Author's contribution: All aspects of this research were performed by Michael J. Williams.

\section{References}

I. Kizhatil K, Wu Y, Sen A, Bennett $\mathrm{V}$ : A new activity of doublecortin in recognition of the phospho-FIGQY tyrosine in the cytoplasmic domain of neurofascin. J Neurosci 2002, 22:7948-7958.

2. Lemaitre B, Hoffmann J: The host defense of Drosophila melanogaster. Annu Rev Immunol 2007, 25:697-743.

3. Williams MJ: Drosophila hemopoiesis and cellular immunity. J Immunol 2007, I 78:47 I I-47|6.

4. Lanot R, Zachary D, Holder F, Meister M: Postembryonic hematopoiesis in Drosophila. Dev Biol 200I, 230:243-257.

5. Sorrentino RP, Carton Y, Govind S: Cellular immune response to parasite infection in the Drosophila lymph gland is developmentally regulated. Dev Biol 2002, 243:65-80.

6. Rizki TM, Rizki RM, Grell EH: A mutant affecting the crystal cells in Drosophila melanogaster. Wilhelm Roux's Arch 1980, 188:91-99.

7. Rizki TM, Rizki RM: Properties of the larval haemocytes of Drosophila melanogaster. Experientia 1980, 36:1223-1226.

8. Bidla G, Dushay MS, Theopold U: Crystal cell rupture after injury in Drosophila requires the JNK pathway, small GTPases and the TNF homolog eiger. J Cell Sci 2007, I 20:1209-1215.

9. Russo J, Dupas S, Frey F, Carton Y, Brehelin M: Insect immunity: Early events in the encapsulation process of parasitoid (Leptopilina boulardi) eggs in resistant and susceptible strains of Drosophila. Parasitology 1996, I | 2:135-142.

10. Williams MJ, Ando I, Hultmark D: Drosophila melanogaster Rac2 is necessary for a proper cellular immune response. Genes Cells 2005, 10:813-823.

II. Russo J, Brehelin M, Carton Y: Haemocyte changes in resistant and susceptible strains of $D$. melanogaster caused by virulent and avirulent strains of the parasitic wasp Leptopilina boulardi. J Insect Physiol 200I, 47:167-172.

12. Rose DM, Alon R, Ginsberg MH: Integrin modulation and signaling in leukocyte adhesion and migration. Immunol Rev 2007, 218:126-134.

13. Cernuda-Morollon E, Ridley AJ: Rho GTPases and leukocyte adhesion receptor expression and function in endothelial cells. Circ Res 2006, 98:757-767.

14. Hammer DA: Leukocyte adhesion: What's the catch? Curr Biol 2005, I5:R96-99.

15. Irving P, Ubeda J, Doucet D, et al.: New insights into Drosophila larval haemocyte functions through genome-wide analysis. Cell Microbiol 2005, 7:335-350.

16. Nardi JB, Zhuang S, Pilas B, Mark Bee C, Kanost MR: Clustering of adhesion receptors following exposure of insect blood cells to foreign surfaces. J Insect Physiol 2005, 5 1:555-564.

17. Nardi JB, Pilas B, Bee CM, Zhuang S, Garsha K, Kanost M: Neuroglian-positive plasmatocytes of Manduca sexta. and the initiation of haemocyte attachment to foreign surfaces. Dev Comp Immunol 2006, 30:447-462.

18. Zhuang S, Kelo L, Nardi JB, Kanost MR: Neuroglian on haemocyte surfaces is involved in homophilic and heterophilic interac- 
tions of the innate immune system of Manduca sexta. Dev Comp Immunol 2007, 3 I: I I59-I I 67.

19. Zhuang S, Kelo L, Nardi JB, Kanost MR: Multiple $\alpha$ subunits of integrin are involved in cell-mediated responses of the Manduca immune system. Dev Comp Immunol 2008, 32:365-379.

20. Prevost N, Woulfe D, Tanaka T, Brass LF: Interactions between eph kinases and ephrins provide a mechanism to support platelet aggregation once cell-to-cell contact has occurred. Proc Natl Acad Sci USA 2002, 99:9219-9224.

21. Bieber AJ, Snow PM, Hortsch M, Patel NH, Jacobs JR, Traquina ZR, Schilling J, Goodman CS: Drosophila neuroglian: A member of the immunoglobulin superfamily with extensive homology to the vertebrate neural adhesion molecule LI. Cell 1989, 59:447-460.

22. Hortsch M, Bieber AJ, Patel NH, Goodman CS: Differential splicing generates a nervous system-specific form of Drosophila neuroglian. Neuron 1990, 4:697-709.

23. Hortsch $M$ : The LI family of neural cell adhesion molecules: Old proteins performing new tricks. Neuron 1996, 17:587-593.

24. Chen L, Ong B, Bennett V: LAD-I, the Caenorhabditis elegans LICAM homologue, participates in embryonic and gonada morphogenesis and is a substrate for fibroblast growth factor receptor pathway-dependent phosphotyrosine-based signaling. J Cell Biol 200I, I 54:84I-855.

25. Nagaraj K, Hortsch M: Phosphorylation of LI-type cell-adhesion molecules-ankyrins away! Trends Biochem Sci 2006 , $31: 544-546$.

26. Jenkins SM, Kizhatil K, Kramarcy NR, Sen A, Sealock R, Bennett V: FIGQY phosphorylation defines discrete populations of $L I$ cell adhesion molecules at sites of cell-cell contact and in migrating neurons. J Cell Sci 200 I, I I 4:3823-3835.

27. Gleeson JG, Allen KM, Fox JW, et al.: Doublecortin, a brain-specific gene mutated in human $X$-linked lissencephaly and double cortex syndrome, encodes a putative signaling protein. Cell 1998, 92:63-72

28. Caspi M, Atlas R, Kantor A, Sapir T, Reiner O: Interaction between LISI and doublecortin, two lissencephaly gene products. Hum Mol Genet 2000, 9:2205-2213.

29. Keays D: Neuronal migration: Unraveling the molecular pathway with humans, mice, and a fungus. Mamm Genome 2007, 1 8:425-30.

30. Keely PJ, Westwick JK, Whitehead IP, Der CJ, Parise LV: Cdc42 and Racl induce integrin-mediated cell motility and invasiveness through PI(3)K. Nature 1997, 390:632-636.

31. Morin X, Daneman R, Zavortink M, Chia W: A protein trap strategy to detect GFP-tagged proteins expressed from their endogenous loci in Drosophila. Proc Natl Acad Sci USA 200I, 98:15050-15055.

32. Yamamoto M, Ueda R, Takahashi K, Saigo K, Uemura T: Control of axonal sprouting and dendrite branching by the nrg-ank complex at the neuron-glia interface. Curr Biol 2006, 16:1678-1683.

33. Kurucz E, Zettervall C, Sinka R, et al.: Hemese, a haemocyte-specific transmembrane protein, affects the cellular immune response in Drosophila. Proc Natl Acad Sci USA 2003, 100:2622-2627.

34. Kurucz E, Markus R, Zsamboki J, et al.: Nimrod, a putative phagocytosis receptor with EGF repeats in Drosophila plasmatocytes. Curr Biol 2007, I 7:649-654.

35. Genova JL, Fehon RG: Neuroglian, gliotactin, and the $\mathbf{N a}+/ \mathbf{K}+$ ATPase are essential for septate junction function in Drosophila. J Cell Biol 2003, I61:979-989.

36. Liu Z, Xie T, Steward R: LisI, The Drosophila homolog of a human lissencephaly disease gene, is required for germline cell division and oocyte differentiation. Development 1999 , 1 26:4477-4488.

37. Liu Z, Steward R, Luo L. Drosophila Lis I is required for neuroblast proliferation, dendritic elaboration and axonal transport. Nature Cell Biol 2000, 2:776-783.

38. Lei Y, Warrior R: The Drosophila Lissencephalyl (DLisI) gene is required for nuclear migration. Dev Biol 2000, 226:57-72.

39. Tanaka T, Serneo FF, Higgins C, Gambello MJ, Wynshaw-Boris A, Gleeson JG: LisI and doublecortin function with dynein to mediate coupling of the nucleus to the centrosome in neuronal migration. / Cell Biol 2004, 165:709-72I.
40. Giot L, Bader JS, Brouwer C, et al:: A protein interaction map of Drosophila melanogaster. Science 2003, 302(565I): 1727-36.

4I. Morris SM, Anaya P, Xiang X, Morris NR, May GS, Yu-Lee L: A prolactin-inducible $T$ cell gene product is structurally similar to the Aspergillus nidulans nuclear movement protein NUDC. Mol Endocrinol 1997, I I:229-236.

42. Williams $M$, Wiklund $M$, Wikman S, Hultmark D: Racl signalling in the Drosophila larval cellular immune response. J Cell Sci 2006, I | 9:20|5-2024.

Publish with Biomed Central and every scientist can read your work free of charge

"BioMed Central will be the most significant development for disseminating the results of biomedical research in our lifetime. "

Sir Paul Nurse, Cancer Research UK

Your research papers will be:

- available free of charge to the entire biomedical community

- peer reviewed and published immediately upon acceptance

- cited in PubMed and archived on PubMed Central

- yours - you keep the copyright 